\title{
Zeno of Elea Shines a New Light on Quantum Weirdness
}

\author{
María Esther Burgos \\ Department of Physics, University of Los Andes, Mérida, Venezuela \\ Email: mburgos25@gmail.com
}

How to cite this paper: Burgos, M.E. (2017) Zeno of Elea Shines a New Light on Quantum Weirdness. Journal of Modern Physics, 8, 1382-1397.

https://doi.org/10.4236/jmp.2017.88087

Received: April 17, 2017

Accepted: July 18, 2017

Published: July 21, 2017

Copyright (C) 2017 by author and Scientific Research Publishing Inc. This work is licensed under the Creative Commons Attribution International License (CC BY 4.0).

http://creativecommons.org/licenses/by/4.0/

\section{(c) (†) Open Access}

\begin{abstract}
After a brief reference to the quantum Zeno effect, a quantum Zeno paradox is formulated. Our starting point is the usual version of Time Dependent Perturbation Theory. Although this theory is supposed to account for transitions between stationary states, we are led to conclude that such transitions cannot occur. Paraphrasing Zeno, they are nothing but illusions. Two solutions to the paradox are introduced. The first as a straightforward application of the postulates of Orthodox Quantum Mechanics; the other is derived from a Spontaneous Projection Approach to quantum mechanics previously formulated. Similarities and differences between both solutions are highlighted. A comparison between the two versions of quantum mechanics, supporting their corresponding solutions to the paradox, shines a new light on quantum weirdness. It is shown, in particular, that the solution obtained in the framework of Orthodox Quantum Mechanics is defective.
\end{abstract}

\section{Keywords}

Quantum Weirdness, Quantum Measurements, Spontaneous Quantum Jumps, Time Dependent Perturbation Theory, Quantum Zeno Paradox

\section{Introduction and Outlook}

The Greek philosopher Zeno of Elea (ca. 490-430 BC) supported Parmenide's doctrine. This philosophy states that, contrary to the evidence of our senses, the belief in plurality and change is mistaken; in particular motion is nothing but an illusion.

The most popular Zeno paradoxes concerning motion are "Achilles and the Tortoise" and the "Arrow Paradox". In the latter it is assumed that for motion to occur, an object must change the position which it occupies. In the case of an arrow in flight, Zeno argues that "the flying arrow is at rest, which result follows from the assumption that time is composed of moments... he says that if every- 
thing when it occupies an equal space is at rest, and if that which is in locomotion is always in a now, the flying arrow is therefore motionless. (Aristotle Physics, 239b. 30) Zeno abolishes motion, saying 'What is in motion moves neither in the place it is nor in one in which it is not'. (Diogenes Laertius Lives of Famous Philosophers, ix.72)" [1].

In 1977 Baidyanath Misra and George Sudarshan studied the behavior of an unstable particle continuously observed to see whether it decays or not [2]. The resulting effect have previously been described by Alan Turing in the following terms: "it is easy to show using standard [quantum] theory that if a system starts in an eigenstate of some observable, and measurements are made of that observable $N$ times a second, then, even if the state is not a stationary one, the probability that the system will be in the same state after, say, one second, tends to one as $N$ tends to infinity; that is, that continual observations will prevent motion..." [3]. Initially this argument received the name of Turing paradox.

In their 1977 paper, Misra and Sudarshan referred to the behavior of a quantum system subjected to frequent ideal measurements. They considered the process of continuing observation as the limiting case of successions of (practically) instantaneous measurements as the intervals between successive measurements approach zero. They argued that, "since there does not seem to be any principle, internal to quantum theory, that forbids the duration of a single measurement or the dead time between successive measurements from being arbitrarily small, the process of continuous observation seems to be an admissible process in quantum theory" [2]. They concluded that an unstable particle which is continuously observed to see whether it decays or not will never be found to decay and named this phenomenon the quantum Zeno paradox [2].

Misra and Sudarshan article stimulated a great deal of theoretical and experimental work. The possibility that the decay of an unstable particle could be prevented by continued observation was, however, considered an alarming result by some physicists. In particular, as early as in 1983, Mario Bunge and Andrés Kálnay explicitly dealt with the suspicion that the quantum Zeno paradox must be a fraud [4] [5] [6].

In 1990 Wayne Itano et al. published a paper entitled Quantum Zeno effect. In its abstract they assert: "The quantum Zeno effect is the inhibition of transitions between quantum states by frequent measurements of the state. The inhibition arises because the measurement causes a collapse (reduction) of the wave function. If the time between measurements is short enough, the wave function usually collapses back to the initial state. We have observed this effect in an $\mathrm{rf}$ transition... Short pulses of light, applied at the same time as the rf field, made the measurements" ([7]; emphases added).

In 2009, Itano published a revision of different opinions regarding the quantum Zeno effect. He acknowledges that "there has been much disagreement as to how the quantum Zeno effect should be defined and as to whether it is really a paradox, requiring new physics, or merely a consequence of 'ordinary' quantum mechanics" [8]. For instance, according to Asher Peres, the quantum Zeno effect 
has nothing paradoxical: "What happens simply is that the quantum system is overwhelmed by the meters which continuously interact with it" ([9], p. 394).

The theoretical and experimental work dealing with the quantum Zeno effect is exciting. But its relation with Zeno's arrow paradox is questionable: Zeno's purpose was not to stop the flying arrow; it was to show that motion is an illusion. By contrast, both Turing's argument and Misra and Sudarshan's contribution aim to stop transitions between quantum states by frequent measurements; let alone the experiment by Itano et al. (and many others we have not mention for brevity) where transitions between quantum states seem to have been truly inhibited, at least partially.

Differing from other references to the quantum Zeno effect, the present paper highlights a True Quantum Zeno paradox (TQZ paradox for short): we show that the usual version of Time Dependent Perturbation Theory (TDPT) leads to the conclusion that transitions between stationary states cannot happen. They are nothing but illusions.

The outlook of this paper is as follows: In Section 2, we formulate the TQZ paradox. In Section 3 we introduce and compare two different solutions to the paradox: an orthodox solution results from a straightforward application of the postulates of Orthodox (Ordinary, Standard) Quantum Mechanics (OQM); the other is derived from a Spontaneous Projection Approach to quantum mechanics (SPA) previously formulated. Section 4 contrasts the main traits of SPA and OQM. In particular, similarities and differences between both solutions to TQZ paradox are highlighted. Section 5 sums up the conclusions of the present work.

\section{Formulation of TQZ Paradox}

The aim of TDPT is to calculate the transition probability between stationary states induced by a time dependent perturbation. In the following we sketch the essential features of TDPT. For more details see for instance: D. R. Bes ([10], Chapter IX); C. Cohen-Tannoudji et al. ([11], Chapter XIII); P. A. M. Dirac ([12], Chapter VII); W. Heitler ([13], Chapter IV); E. Merzbacher ([14], Chapter XIX); and/or A. Messiah ([15], Chapitre XVII). Notes. Symbols used by these authors may have been changed for homogeneity. All the states referred to in this paper are normalized.

Consider a system with Hamiltonian $\mathscr{E}$ which does not depend explicitly on time. It will be called the unperturbed Hamiltonian of the system. Its eigenvalue equations are

$$
\mathcal{E}\left|\phi_{n}\right\rangle=E_{n}\left|\phi_{n}\right\rangle
$$

where $E_{n}(n=1,2, \cdots)$ are the eigenvalues of $\mathscr{E}$ and $\left|\phi_{n}\right\rangle$ the corresponding eigenstates. For simplicity we assume $\mathcal{E}$ spectrum to be entirely discrete and non-degenerate.

We shall suppose that at initial time $t=0$ the system is in the stationary state $\left|\phi_{j}\right\rangle$. If for $t \geq 0$ the Hamiltonian were $\mathcal{E}$, the state vector at time $\mathrm{t}$ would be

$$
|\psi(t)\rangle=\mathrm{e}^{-i E_{j} t / \hbar}|\psi(0)\rangle=\mathrm{e}^{-i E_{j} t / \hbar}\left|\phi_{j}\right\rangle
$$


where $\hbar$ is Planck's constant divided by $2 \pi$ and $i$ is the imaginary unity. The kets $|\psi(t)\rangle$ and $\left|\phi_{j}\right\rangle$ differ only by the global phase factor $\mathrm{e}^{-i E_{j} t / \hbar}$. So all the kets $|\psi(t)\rangle$ given by Equation (2) represent one and the same eigenstate corresponding to the eigenvalue $E_{j}$.

A system in a stationary state (i.e. an eigenstate of the unperturbed Hamiltonian $\mathscr{E})$ will remain in that state forever. Nevertheless, TDPT establishes that by applying a time dependent perturbation, transitions between different eigenstates of $\varepsilon$ can be induced and determines the probability corresponding to every particular transition.

If at $t=0$ a time dependent perturbation $\mathscr{W}(t)$ is applied, for $t \geq 0$ the total, perturbed Hamiltonian will be

$$
\mathscr{H}(t)=\mathcal{E}+\mathscr{W}(t)
$$

The perturbation $\mathscr{W}(t)$ causes the state $|\psi(0)\rangle$ to change. According to TDPT, the Schrödinger evolution leads the initial state $|\psi(0)\rangle=\left|\phi_{j}\right\rangle$ to the state

$$
|\psi(t)\rangle=\psi_{\varpi}(t)=\mathcal{U}(t, 0)|\psi(0)\rangle=\mathcal{C}(t, 0)\left|\phi_{j}\right\rangle
$$

where $\mathcal{U}(t, 0)$ is, by definition, the evolution operator corresponding to the Hamiltonian $\mathscr{H}(t)$. We have written $|\psi(t)\rangle=\left|\psi_{w}(t)\right\rangle$ to stress that the state vector depends on the perturbation $\mathscr{U}(t)$ through the evolution operator थ $(t, 0)$.

Let us underline the difference between the state vector at time $t$ when no time dependent perturbation is applied and the state vector at time $t$ resulting from the application of $\omega(t)$ during the time interval $(0, t)$. In the former case, the state vector coincides with the stationary state $\left|\phi_{j}\right\rangle$; see Equation (2). In the latter, the state vector will in general not be stationary but a linear superposition of several (at least two) stationary states; one of them being $\left|\phi_{j}\right\rangle$.

At this point the probability of a transition taking place from state $\left|\phi_{j}\right\rangle$ to state $\left|\phi_{k}\right\rangle$ during the time interval $(0, t)$ is introduced. In the words of Paul Dirac, "at time $t$ the ket corresponding to the state in Schrödinger's picture will be $\mathcal{U}(t, 0)\left|\phi_{j}\right\rangle$ according to Equation (4). The probability of the $E_{n}$ 's then having the values $E_{k}$ is

$$
\mathscr{P}_{0, t}\left(E_{j} E_{k}\right)=\left|\left\langle\phi_{k}|\mathcal{U}(t, 0)| \phi_{j}\right\rangle\right|^{2}
$$

For $k \neq j, \mathscr{P}_{0, t}\left(E_{j} E_{k}\right)$ is the probability of a transition taking place from state $\left|\phi_{j}\right\rangle$ to state $\left|\phi_{k}\right\rangle$ during the time interval $(0, t)$, while $\mathscr{P}_{0, t}\left(E_{j} E_{j}\right)$ is the probability of no transition taking place at all. The sum of $\mathscr{P}_{0, t}\left(E_{j} E_{k}\right)$ for all $k$ is, of course, unity" ([12], pp. 172-173; emphases added).

TDPT deals with processes having two clearly different stages [16]. In the first-during the time interval $(0, t)-$ a Schrödinger evolution leads the state vector from $|\psi(0)\rangle$ to $|\psi(t)\rangle$ given by Equation (4) with certitude. In the second an instantaneous projection of $|\psi(t)\rangle$ to a stationary state $\left|\phi_{k}\right\rangle$ is ruled by probability laws [16]. $|\psi(t)\rangle$ can collapse either to a state $\left|\phi_{k}\right\rangle$ where $k \neq j$, or to the initial state $\left|\phi_{j}\right\rangle$. According to Dirac, in this last case no transi- 
tion takes place at all. This does not mean that the system stays in the initial state $\left|\phi_{j}\right\rangle$ during the whole process. It means: during the interval $(0, t)$ the system follows a Schrödinger evolution and at instant $t$, when the state vector is $|\psi(t)\rangle$ given by Equation (4), it jumps to $\left|\phi_{j}\right\rangle$.

A collapse at $t$ implies that the process is discontinuous at this instant. Since the sum of probabilities of a transition from $\left|\phi_{j}\right\rangle$ to $\left|\phi_{k}\right\rangle$ with $k \neq j$, plus the probability of no transition takes place at all during the time interval $(0, t)$ is

$$
\sum_{k \neq j} \mathscr{P}_{0, t}\left(E_{j} E_{k}\right)+\mathscr{P}_{0, t}\left(E_{j} E_{j}\right)=1
$$

there is no room for a non-null probability corresponding to a process continuous at time $t$.

Let us now consider the following argument:

(a) A system initially in the state $|\psi(0)\rangle=\left|\phi_{j}\right\rangle$ follows a Schrödinger evolution during $(0, t)$. For that to happen, the state vector must be continuous at every instant of this interval. Under these conditions $|\psi(t)\rangle=\mathcal{U}(t, 0)\left|\phi_{j}\right\rangle$ with certitude.

(b) The interval $(0, t)$ can be divided into the intervals $\left(0, t^{\prime}\right)$ and $\left(t^{\prime}, t\right)$, where $0<t^{\prime}<t$. If the initial state is $\left|\phi_{j}\right\rangle$, a Schrödinger evolution during the interval $\left(0, t^{\prime}\right)$ leads the initial state to $\left|\psi\left(t^{\prime}\right)\right\rangle=\mathcal{U}\left(t^{\prime}, 0\right)\left|\phi_{j}\right\rangle$ with certitude.

(c) Taking into account the validity of Equations (5) and (6) during the interval $\left(0, t^{\prime}\right)$ we are forced to conclude that the probability corresponding to a process continuous at time $t^{\prime}$ is null. But if the state vector is not continuous at t' the system cannot follow a Schrödinger evolution during the interval $(0, t)$. This conclusion contradicts (a).

With the noticeable exception of Albert Messiah, neither Dirac nor any other author known to us imposes any particular condition on the interval $(0, t)$; the condition imposed by Messiah will be discussed in the next section. In the usual version of TDPT it is assumed that Equations (5) and (6) are valid during every time interval $(0, t)$. The resulting contradiction is TQZ paradox.

Let us review what happens in a small time interval $(0, d t)$. The infinitesimal evolution operator between 0 and $d t$ can be written

$$
\mathcal{X}(d t, 0)=\mathscr{Y}-\frac{i}{\hbar} \mathscr{H}(0) d t=\mathscr{Y}-\frac{i}{\hbar}[\mathcal{E}+\mathscr{W}(0)] d t
$$

where $\mathscr{G}$ is the identity operator ([11], p. 309). Should the system follow a Schrödinger evolution during the interval $(0, d t)$, the operator $\varkappa(d t, 0)$ would lead it from $\left|\phi_{j}\right\rangle$ to

$$
|\psi(d t)\rangle=\left\{\varsubsetneqq-\frac{i}{\hbar}[\mathcal{E}+\mathscr{\omega}(0)] d t\right\}\left|\phi_{j}\right\rangle
$$

For $k \neq j$ the probability of a transition taking place from state $\left|\phi_{j}\right\rangle$ to state $\left|\phi_{k}\right\rangle$ during the time interval $(0, d t)$ would be

$$
\mathscr{P}_{0, d t}\left(E_{j} E_{k}\right)=\left|\left\langle\phi_{k}|\mathcal{U}(d t, 0)| \phi_{j}\right\rangle\right|^{2}=\left|\left\langle\phi_{k}|\omega(0)| \phi_{j}\right\rangle\right|^{2} \frac{(d t)^{2}}{\hbar^{2}}
$$

the sum of these probabilities for all $k \neq j$ would be 


$$
\sum_{k \neq j} \mathscr{P}_{0, d t}\left(E_{j} E_{k}\right)=\sum_{k \neq j}\left|\left\langle\phi_{k}|\omega(0)| \phi_{j}\right\rangle\right|^{2} \frac{(d t)^{2}}{\hbar^{2}}
$$

and the probability of no transition taking place at all would be

$$
\mathscr{P}_{0, d t}\left(E_{j} E_{j}\right)=1-\sum_{k \neq j}\left|\left\langle\phi_{k}|\%(0)| \phi_{j}\right\rangle\right|^{2} \frac{(d t)^{2}}{\hbar^{2}}
$$

Always assuming that the process is a Schrödinger evolution during the interval $(0, d t)$, we see that if $d t \rightarrow 0$ the probability for a transition between different stationary states becomes negligible while the probability for no transition taking place at all approaches unity. In colloquial speech we would conclude: as soon as the state vector becomes different from the initial state $\left|\phi_{j}\right\rangle$ a projection forces it to return to the starting point.

We have shown, nevertheless, that according to the usual version of TDPT the system cannot follow a Schrödinger evolution during any time interval. Therefore, the state vector at time $t$ cannot be $\mathcal{\chi}(t, 0)\left|\phi_{j}\right\rangle$ and transitions between stationary states during the time interval $(0, t)$ as referred to in TDPT cannot take place. Paraphrasing Zeno, these kinds of transitions between stationary states are nothing but an illusion.

\section{Solving TQZ Paradox}

First solution: While remaining in the framework of OQM, Messiah version of TDPT differs somewhat from the usual one. In his words: "Supposons qu'à l'instant initial $t=0$ le système se trouve dans l'un des états propres de $\mathcal{E}$, l'état $\left|\phi_{j}\right\rangle$ par exemple. Nous nous proposons de calculer la probabilité de le trouver à l'instant $t$ dans un autre état propre de $\mathcal{E}$, l'état $\left|\phi_{k}\right\rangle$ par exemple, dans Péventualité d’une mesure à cet instant. Soit $\mathscr{P}_{0, t}\left(E_{j} E_{k}\right)$ cette quantité, c'est par définition la probabilité de transition de $\left|\phi_{j}\right\rangle$ en $\left|\phi_{k}\right\rangle$ ” ([15], p. 621; emphases added).

In Section 2 we pointed out that, except Messiah, neither Dirac nor any other author known to us imposes any particular condition on the interval $(0, t)$. This is why we could say that both Equation (5) and Equation (6) are valid during every interval $(0, t)$. By contrast, Messiah imposes the condition that an instantaneous measurement be performed at time $t$, as demanded by a straightforward application of the postulates of OQM. If this condition is not fulfilled, the Schrödinger evolution follows and the probability of a transition taking place from $\left|\phi_{j}\right\rangle$ to $\left|\phi_{k}\right\rangle$ during the interval $(0, t)$ is null. The same applies to the sum of probabilities appearing at the 1.h. of Equation (6): it is not unity but 0 .

Even if the notion of instantaneous measurement is questionable ([5]; [17], p. 200), Messiah successfully eludes TQZ paradox through this concept. We shall call it the orthodox solution to TQZ paradox. Figure 1 illustrates the case where the non-perturbed Hamiltonian $\mathcal{E}$ has only two eigenvalues: $E_{j}$ and $E_{k}$; we suppose both of them non-degenerate. In the first stage no measurement is performed. As a consequence, during the time interval $(0, t)$ a Schrödinger evolution 


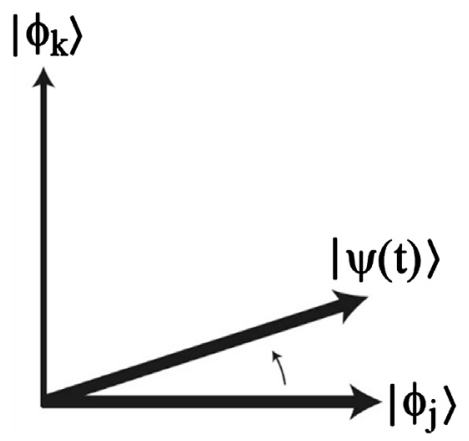

(a)

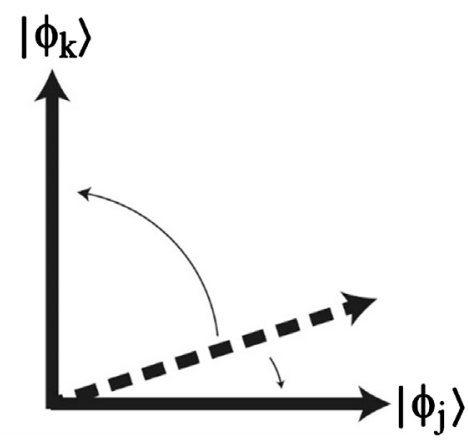

(b)

Figure 1. The Orthodox Solution to TQZ paradox: (a) During the first stage a Schrödinger evolution leads the state vector from $|\psi(0)\rangle=\left|\phi_{j}\right\rangle$ to $|\psi(t)\rangle=\mathscr{U}(t, 0)\left|\phi_{j}\right\rangle$. (b) In the second stage an instantaneous measurement is performed. As a consequence $|\psi(t)\rangle$ jumps either to $\left|\phi_{k}\right\rangle$ or to $\left|\phi_{j}\right\rangle$.

leads the state vector from $|\psi(0)\rangle=\left|\phi_{j}\right\rangle$ to $|\psi(t)\rangle=\mathscr{U}(t, 0)\left|\phi_{j}\right\rangle$ with certitude. In the second stage an instantaneous measurement is performed. It is worth noting that $|\psi(t)\rangle$ cannot remain continuous at time $t$. It jumps either to $\left|\phi_{k}\right\rangle$ with probability $\left|\left\langle\phi_{k} \mid \psi(t)\right\rangle\right|^{2}$; or to $\left|\phi_{j}\right\rangle$ with probability $\left|\left\langle\phi_{j} \mid \psi(t)\right\rangle\right|^{2}$.

Second solution: SPA provides another solution to TQZ paradox. According to this approach [18] [19] [20]:

(i) Two kinds of processes, irreducible to one another, occur in nature: the strictly continuous and causal ones; and those implying discontinuities, where the system's state $|\psi(t)\rangle$ collapses. (ii) The system's state $|\psi(t)\rangle$ has the tendency to jump (collapse, be projected, be reduced) to the eigenstates of operators representing conserved physical quantities. (iii) A postulate ensures that projections of $|\psi(t)\rangle$ are possible only in cases where they do not violate the statistical sense of conservation laws. The compromise between the tendency $|\psi(t)\rangle$ has to jump to the eigenstates of operators representing conserved physical quantities and the limitations imposed by the postulate ensuring the statistical sense of conservation laws is at the basis of the definition of preferential states. (iv) If a system in the state $|\psi(t)\rangle$ with Hamiltonian $\mathcal{E}$ (which does not depend explicitly on time) has the preferential states $\left|\varphi_{q}\right\rangle(q=1,2, \cdots)$, then:

$$
|\psi(t)\rangle=\sum_{q} \gamma_{q}(t)\left|\varphi_{q}\right\rangle
$$

where $\gamma_{q}(t)=\left\langle\varphi_{q} \mid \psi(t)\right\rangle \neq 0$. (v) If the Hamiltonian is $\mathscr{H}(t)=\mathcal{E}+\mathscr{W}(t)$ (where $\mathscr{W}(t)$ depends explicitly on time), the preferential states of the system in the state $|\psi(t)\rangle$ coincide with those the system has for $\omega(t)=0$. (vi) If $|\psi(t)\rangle$ does not have preferential states, the Schrödinger evolution follows. By contrast, if the preferential states are $\left|\varphi_{1}\right\rangle,\left|\varphi_{2}\right\rangle, \cdots$, during the small interval $(t, t+d t)$ the state $|\psi(t)\rangle$ can undergo the following changes

$$
|\psi(t)\rangle \rightarrow|\psi(t+d t)\rangle=\left|\varphi_{q}\right\rangle
$$

with probability 


$$
d \mathscr{P}_{q}(t)=\left|\gamma_{q}(t)\right|^{2} \frac{d t}{\tau(t)}
$$

or

$$
|\psi(t)\rangle \rightarrow\left|\psi_{\mathcal{u}}(t+d t)\right\rangle=\mathscr{U}(t+d t, t)|\psi(t)\rangle
$$

with probability

$$
d \mathscr{P}_{\mathscr{C}}(t)=1-\frac{d t}{\tau(t)}
$$

Here

$$
\tau(t) \Delta \varepsilon(t)=\frac{\hbar}{2}
$$

and

$$
[\Delta \mathcal{E}(t)]^{2}=\left\langle\psi(t)\left|\mathcal{E}^{2}\right| \psi(t)\right\rangle-[\langle\psi(t)|\mathcal{E}| \psi(t)\rangle]^{2}
$$

Changes (13) are projections to one of the preferential states with probabilities given by Equation (14). As $|\psi(t)\rangle$ is normalized, the sum of probabilities for jumps like $|\psi(t)\rangle \rightarrow\left|\varphi_{1}\right\rangle$, or $|\psi(t)\rangle \rightarrow\left|\varphi_{2}\right\rangle$, etc., to occur is $\frac{d t}{\tau(t)}$. The change (15) is a Schrödinger evolution with probability given by Equation (16). The sum of probabilities corresponding to all the possibilities is

$$
\sum_{q} d \mathscr{P}_{q}(t)+d \mathscr{P}_{\mathscr{C}}(t)=1
$$

Except (v), all these points have been introduced and discussed in previous papers [18] [19] [20]. For more details on points (ii) and (iii) leading to the definition of preferential states see Appendix A; for examples of the determination of preferential states see Appendix B.

For simplicity we assume $\mathscr{E}$ spectrum to be entirely discrete and non-degenerate. The state vector can be written

$$
|\psi(t)\rangle=\sum_{k} c_{k}(t)\left|\phi_{k}\right\rangle
$$

where $c_{k}(t)=\left\langle\phi_{k} \mid \psi(t)\right\rangle$. At $t=0,|\psi(0)\rangle=\left|\phi_{j}\right\rangle, \quad c_{j}(0)=1$ and $c_{k}(0)=0$ for $k \neq j$. The perturbation $\mathscr{\omega}(t)$ causes $|\psi(0)\rangle$ to change: for $t>0$, $\left|c_{j}(t)\right|$ decreases and at least one of the $c_{k}(t)$ becomes non-null. Even if not every stationary state is a preferential state, and not every preferential state is a stationary state [18], in the particular case where $\mathscr{E}$ has discrete non-degenerate spectrum, every preferential state is a stationary state; see Appendix B.

If $d t \ll \tau(t)$, the dominant process is the Schrödinger evolution [18]. As soon as the condition $c_{k}(t) \neq 0$ is fulfilled for $k \neq j$ the corresponding $\left|\phi_{k}\right\rangle$ becomes a preferential state of the system in the state $|\psi(t)\rangle$ and a transition from $\left|\phi_{j}\right\rangle$ to $\left|\phi_{k}\right\rangle$ during the time interval $(0, t)$ is possible. Hence the system does not remain stuck to the initial state $\left|\phi_{j}\right\rangle$. This is the SPA solution to TQZ paradox. 
Figure 2 illustrates SPA solution in the case where the non-perturbed Hamiltonian $\mathcal{E}$ has only two eigenvalues: $E_{j}$ and $E_{k}$; we suppose both of them nondegenerate. If no spontaneous projection happens in the time interval $(0, t)$ a Schrödinger evolution leads the state vector from $|\psi(0)\rangle=\left|\phi_{j}\right\rangle$ to $|\psi(t)\rangle=\mathscr{U}(t, 0)\left|\phi_{j}\right\rangle$. During the small interval $(t, t+d t)$ the state $|\psi(t)\rangle$ can undergo the following changes: either it jumps to one of its preferential states $\left(\left|\phi_{k}\right\rangle\right.$ and $\left.\left|\phi_{j}\right\rangle\right)$, or it follows a Schrödinger evolution which leads it from $|\psi(t)\rangle$ to $|\psi(t+d t)\rangle=\mathcal{U}(t+d t, t)|\psi(t)\rangle$. The probabilities are, respectively,

$$
d \mathscr{P}_{k}(t)=\left|\left\langle\phi_{k} \mid \psi(t)\right\rangle\right|^{2} \frac{d t}{\tau(t)} ; d \mathscr{P}_{j}(t)=\left|\left\langle\phi_{j} \mid \psi(t)\right\rangle\right|^{2} \frac{d t}{\tau(t)} \text {; and } d \mathscr{P}_{\mathfrak{U}}(t)=1-\frac{d t}{\tau(t)} \text {. }
$$

Differing from what happens in the framework of OQM there is always room for a Schrödinger evolution in SPA. There is, however, a complete agreement between SPA solution and orthodox solution to TQZ paradox in which concerns the ratio of probabilities corresponding to jumps to $\left|\phi_{k}\right\rangle$ and to $\left|\phi_{j}\right\rangle$ : in both cases it takes on the value $\frac{\left|\left\langle\phi_{k} \mid \psi(t)\right\rangle\right|^{2}}{\left|\left\langle\phi_{j} \mid \psi(t)\right\rangle\right|^{2}}$.

\section{SPA versus OQM}

OQM was first formulated by Dirac in 1930 [12]. It refers to individual systems and imposes two laws of change of the state vector $|\psi(t)\rangle$. Spontaneous processes are governed by the Schrödinger equation, a deterministic law. Measurement processes are ruled by probability laws through Born's postulate and the projection postulate. Measurement processes require either the intervention of an observer or the interaction of the quantum system with a macroscopic object playing the role of measuring device [20]. It has been pointed out that the projection postulate introduces a subjective element into the theory; it

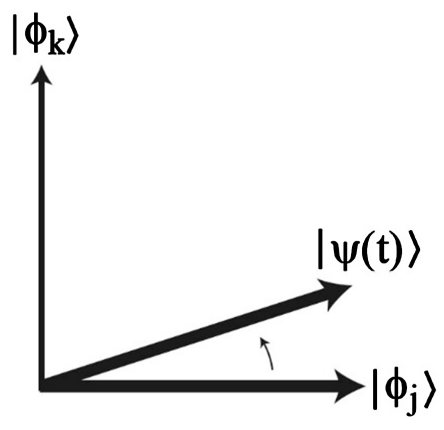

(a)

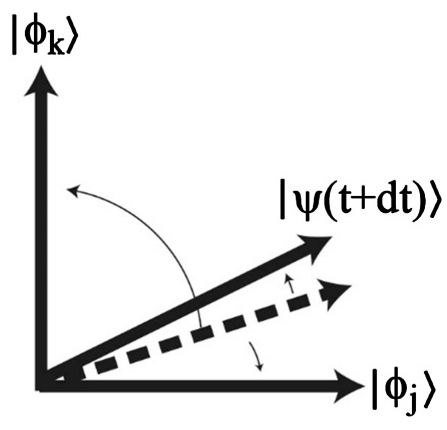

(b)

Figure 2. SPA Solution to TQZ paradox: (a) If no spontaneous projection happens during the time interval $(0, t)$, a Schrödinger evolution leads the state vector from $|\psi(0)\rangle=\left|\phi_{j}\right\rangle$ to $|\psi(t)\rangle=\mathcal{U}(t, 0)\left|\phi_{j}\right\rangle$. (b) In the small interval $(t, t+d t)$ the system's state $|\psi(t)\rangle$ has three possibilities: either it follows a Schrödinger evolution which leads it to $|\psi(t+d t)\rangle=\mathcal{C}(t+d t, t)|\psi(t)\rangle$, or it jumps to one of its preferential states: $\left|\phi_{k}\right\rangle$ and $\left|\phi_{j}\right\rangle$. 
conflict with the Schrödinger equation; and it implies a kind of action-at-a-distance ([17], pp. 191-205; [20]).

The presence in parallel of two different, irreducible to one another laws accounting for the change of the state vector $|\psi(t)\rangle$ calls for a rule to decide which one should be applied in every particular case. But OQM does not provide such a rule. Concerning this issue John Bell complains: “during 'measurement' the linear Schrödinger evolution is suspended and an ill-defined 'wave-function collapse' takes over. There is nothing in the mathematics to tell what is 'system' and what is 'apparatus,' nothing to tell which natural processes have the special status of 'measurements.' Discretion and good taste, born from experience, allow us to use quantum theory with marvelous success, despite the ambiguity of the concepts named above in quotation marks" ([21], p.160; emphasis added).

OQM marvelous success in the area of experimental predictions is mostly based on TDPT. It is agreed that the method provided by this theory must be used to solve all problems involving time, including time dependent spontaneous processes. Should TDPT be discarded, OQM and many of its extensions would lose almost completely their power of explanation and prediction [16]. At the same time, TDPT is a good example of the ambiguities OQM confronts. In Section 2 we pointed out that TDPT deals with processes having two clearly different stages [16]. In the first-during the time interval $(0, t)-$ a Schrödinger evolution leads the system's state from $|\psi(0)\rangle=\left|\phi_{j}\right\rangle$ to $|\psi(t)\rangle=\mathscr{C}(t, 0)\left|\phi_{j}\right\rangle$. In the second an instantaneous projection of $|\psi(t)\rangle$ to a stationary state $\left|\phi_{k}\right\rangle$ is ruled by probability laws [16]. Both laws are necessary for TDPT to work, but the fact that TDPT requires the application of postulates concerning measurements to account for processes supposedly spontaneous is at the very heart of OQM incoherence [16].

SPA, a version of quantum mechanics previously introduced [18] [19] [20], deals with these issues. Our motivation to formulate this approach is the restoration of philosophical realism as the basis of quantum mechanics. Albert Einstein was right when he proclaimed: "the belief in an external world independent of the perceiving subject is the basis of all natural science" [22]. We have also taken into account Bunge's notion of epistemological realism: "The main epistemological problem about quantum theory is whether it represents real (autonomously existing) things, and therefore whether it is compatible with epistemological realism. The latter is the family of epistemologies which assume that (a) the world exists independently of the knowing subject, and (b) the task of science is to produce maximally true conceptual models of reality...” ([17], pp. 191-192).

Other approaches aiming to confront quantum weirdness are close to, but different from OQM. By contrast, SPA does not introduce substantial changes into the theory. It does not modify the Schrödinger equation and recovers a version of Born's postulate where no reference to measurement is made. The exponential decay law is obtained in cases where the Hamiltonian does not depend explicitly on time [18]. Differing from OQM, SPA yields an expression for the probability of transitions to the continuum which is valid for every time and, except for some minimal restrictions, for every added potential. This prediction 
could be tested by experiment [19].

It has been pointed out that some theories of spontaneous state reduction are incompatible with the attainment of equilibrium [23]. This is obviously not the case of SPA where stationary states are not only possible: they play a fundamental role. We should also stress the radical difference between SPA and theories of quantum measurement based in the concept of decoherence. According to these theories, the off-diagonal elements of the density matrix should progressively vanish; it is not clear, however, why all diagonal elements but one should vanish [24]. By contrast, SPA states that a spontaneous projection to a preferential state instantaneously deletes as well the off-diagonal elements of the density matrix as all diagonal elements but one, as established by OQM when a measurement is performed.

The orthodox solution to TQZ paradox obtained in Section 3 results from a straightforward application of the postulates of OQM. But let us perform a close examination of this solution in the particular case where the perturbation applied at $t=0$ does not depend explicitly on time. For $t<0$ the Hamiltonian representing the energy of the system is $\mathfrak{E}$. The corresponding eigenvalue equations are

$$
\mathcal{E}\left|\phi_{n}\right\rangle=E_{n}\left|\phi_{n}\right\rangle
$$

where $E_{n}(n=1,2, \cdots)$ are the eigenvalues of $\mathscr{E}$ and $\left|\phi_{n}\right\rangle$ the corresponding eigenstates. For $t \geq 0$ the Hamiltonian representing the energy of the system is $\mathscr{K}=\mathscr{E}+\mathscr{W}$. The corresponding eigenvalue equations are

$$
\mathscr{K}\left|\xi_{m}\right\rangle=E_{m}^{\prime}\left|\xi_{m}\right\rangle
$$

where $E_{m}^{\prime}(m=1,2, \cdots)$ are the eigenvalues of $\mathscr{K}$ and $\left|\xi_{m}\right\rangle$ the corresponding eigenstates. For simplicity we assume both $\mathcal{E}$ and $\mathscr{K}$ spectra to be entirely discrete and non-degenerate.

According to the postulates of OQM the only possible result of the measurement of a physical quantity is one of the eigenvalues of the operator which represents it. So a measurement of the energy performed at $t \geq 0$ must yield one of the eigenvalues of $\mathscr{K}$, v.g. $E_{r}^{\prime}$. As a consequence, $|\psi(t)\rangle$ must jump to $\left|\xi_{r}\right\rangle$, which is not an eigenstate of $\mathcal{E}$ but an eigenstate of $\mathscr{K}$ [19]. Hence, even if the orthodox solution to TQZ paradox allows us to conclude that the system does not remain stuck to the initial eigenstate $\left|\phi_{j}\right\rangle$, it does not follow that the system performs transitions between eigenstates of the non-perturbed Hamiltonian $\mathcal{E}$. In this sense, this solution to TQZ paradox is defective. It is not evident that this defect will disappear in cases where the perturbation applied at $t=0$ depends explicitly on time.

By contrast, SPA solution to TQZ paradox makes no reference to measurements. Transitions between eigenstates of $\mathcal{E}$ are possible as long as these eigenstates are preferential states of the system in the state $|\psi(t)\rangle$. The preferential states do not change when a perturbation $\%(t)$ which depends explicitly on time is applied (see Section 3). Nevertheless, if the perturbation is constant in time, the preferential states may change. 


\section{Conclusions}

In the framework of OQM, there are no projections without measurements. So it is necessary to invoke measurements even in spontaneous processes where measurements should obviously be absent. This is v.g. the case of absorption and emission of light and of processes occurring in semiconductors.

Both our Critical Review of TDPT [16] and the orthodox solution to TQZ paradox introduced in Section 3 highlight that in OQM the notion of measurement and consequent projections are ad-hoc. By contrast, in SPA projections are not surreptitious but explicitly included in the formalism. The same is true of the rule necessary to decide whether the system will forcibly follow a Schrödinger evolution or not. This is why SPA enjoys of a coherence which is absent from OQM [16] and allows us to provide a satisfactory solution to the True Quantum Zeno Paradox.

\section{Acknowledgements}

We are indebted to Professor J. C. Centeno for many fruitful discussions. We are grateful to Professors F. G. Criscuolo and Marco Ortiz Palanques for some useful comments. We thank Carlos Valero for his assistance with the figures and the transcription of formulas into Math Type.

\section{References}

[1] Huggett, N. (2010) Zeno's Paradoxes. In: Zalta, E.N., Ed., The Stanford Encyclopedia of Philosophy (Winter 2010 Edition). Stanford Encyclopedia of Philosophy, Metaphysics Research Lab, Stanford University; 3.3 The Arrow.

https://plato.stanford.edu/entries/paradox-zeno

[2] Misra, B. and Sudarshan, E.C.G. (1977) Journal of Mathematical Physics, 18, 756763. https://doi.org/10.1063/1.523304

[3] Teuscher, C. and Hofstadter, D. (2004) Alan Turing: Life and Legacy of a Great Thinker. Springer, p. 54. https://doi.org/10.1007/978-3-662-05642-4

[4] Bunge, M. and Kálnay, A.J. (1983) Il NuovoCimento, 77B, 1-9. https://doi.org/10.1007/bf02738413

[5] Bunge, M. and Kálnay, A.J. (1983) Il NuovoCimento, 77B, 10-18. https://doi.org/10.1007/bf02738414

[6] Maddox, J. (1983) Nature, 306, 111. https://doi.org/10.1038/306111a0

[7] Itano, W.M., Heinzen, D.J., Bollinger, J.J. and Wineland, D.J. (1990) Physical Review A, 41, 2295-2300. https://doi.org/10.1103/PhysRevA.41.2295

[8] Itano, W.M. (2009) Journal of Physics: Conference Series, 196, 012018. https://doi.org/10.1088/1742-6596/196/1/012018

[9] Peres, A. (1993) Quantum Theory: Concepts and Methods. Kluwer Academic Publishers, Dordrecht-Boston-London.

[10] Bes, D.R. (2004) Quantum Mechanics. Springer-Verlag, Berlin-Heidelberg. https://doi.org/10.1007/978-3-662-05384-3

[11] Cohen-Tannoudji, C., Diu, B. and Laloë, F. (1977) Quantum Mechanics. John Wiley \& Sons, New York-London-Sydney-Toronto.

[12] Dirac, P.A.M. (1958) The Principles of Quantum Mechanics. Clarendon Press, Oxford. 
[13] Heitler, W. (1984) The Quantum Theory of Radiation. Dover Publications Inc., New York.

[14] Merzbacher, E. (1961) Quantum Mechanics. John Wiley \& Sons, New York.

[15] Messiah, A. (1965) Mécanique Quantique. Dunod, Paris.

[16] Burgos, M.E. (2016) Journal of Modern Physics, 7, 1449-1454. https://doi.org/10.4236/jmp.2016.712132

[17] Bunge, M. (1985) Treatise on Basic Philosophy, Vol. 7, Philosophy of Science \& Technology. D. Reidel Publishing Company, Dordrecht-Boston-Lancaster.

[18] Burgos, M.E. (1998) Foundations of Physics, 28, 1323-1346. https://doi.org/10.1023/A:1018826910348

[19] Burgos, M.E. (2008) Foundations of Physics, 38, 883-907. https://doi.org/10.1007/s10701-008-9213-5

[20] Burgos, M.E. (2015) The Measurement Problem in Quantum Mechanics Revisited. In: Pahlavani, M., Ed., Selected Topics in Applications of Quantum Mechanics, INTECH, Croatia, 137-173. https://doi.org/10.5772/59209

[21] Bell, M., Gottfried, K. and Veltman, M. (2001) John S. Bell on the Foundations of Quantum Mechanics. Word Scientific, Singapore.

[22] Einstein, A. (1931) James Clerk Maxwell: A Commemoration Volume. Cambridge University Press, Cambridge.

[23] Ballentine, L.E. (1991) Physical Review A, 43, 9-12. https://doi.org/10.1103/PhysRevA.43.9

[24] Laloë, F. (2001) American Journal of Physics, 69, 655-701. https://doi.org/10.1119/1.1356698

[25] Burgos, M.E. (1994) Physics Essays, 7, 69-71. https://doi.org/10.4006/1.3029115

[26] Burgos, M.E. (1997) Speculations in Science and Technology, 20, 183-187.

[27] Burgos, M.E., Criscuolo, F.G. and Etter, T. (1999) Speculations in Science and Technology, 21, 227-233. https://doi.org/10.1023/A:1005552504638

[28] Criscuolo, F.G. and Burgos, M.E. (2000) Physics Essays, 13, 80-84. https://doi.org/10.4006/1.3025430

[29] Burgos, M.E. (2010) Journal of Modern Physics, 1, 137-142. https://doi.org/10.4236/jmp.2010.12019 


\section{Appendix A: The Concept of Preferential States}

Let $\mathscr{Q}$ be the self-adjoint operator representing the physical quantity $\mathcal{A}$ referred to the physical system $\delta$. We assume that the Hamiltonian, denoted by $\mathcal{E}$, does not depend explicitly on time $t$. If $\mathscr{Q}$ fulfills the conditions

$$
\frac{\partial \mathfrak{Q}}{\partial t}=0
$$

and

$$
[\mathfrak{Q}, \mathcal{E}]=0
$$

the state vector $|\psi(t)\rangle$ which represents the state of s has the tendency to jump to the eigenstates of $\mathbb{Q}$. We have shown, however, that this tendency is seldom realized [18] [19] [20].

In addition to the problems referred to in Section 4, OQM conflicts with conservation laws. Let us briefly review this issue which has been largely ignored [25] [26] [27] [28] [29]. By definition the mean value (also called expectation value) of $A$ is

$$
\langle\mathscr{Q}\rangle(t)=\langle\psi(t)|\mathfrak{Q}| \psi(t)\rangle
$$

In Schrödinger evolutions the validity of Equation (A1) and (A2) ensures that $\langle\mathscr{Q}\rangle(t)$ remains a constant in time for every state $|\psi(t)\rangle$. It is said that $\mathscr{Q}$ is a constant of the motion and that the physical quantity $\triangle$ is conserved. By contrast, in processes ruled by another, different law from Schrödinger equation, the validity of Equation (A1) and (A2) does not guarantee that $\langle\mathscr{Q}\rangle(t)$ remains a constant in time. Hence the assertions " $\mathscr{Q}$ is a constant of the motion" and " $A$ is conserved" are not justified [29].

It has been shown that in processes involving projections (like OQM measurement processes) the mean value $\langle\mathfrak{Q}\rangle(t)$ may change even if Equation (A1) and (A2) are fulfilled [20] [28]; this is the case of a process starting at $t_{0}$ and ending at $t_{f}$ which yields $\langle\mathfrak{Q}\rangle\left(t_{f}\right) \neq\langle\mathfrak{Q}\rangle\left(t_{0}\right)$ [29]. Nevertheless, the average of the changes $\delta\langle\mathfrak{Q}\rangle=\langle\mathscr{Q}\rangle\left(t_{f}\right)-\langle\mathfrak{Q}\rangle\left(t_{0}\right)$ obtained by repeating the process many times is close to zero [29]. These remarks justify the adoption of a postulate ensuring the statistical sense of conservation laws [18] [19] [20].

Let us consider a set of $\mathcal{\&}$ orthonormal vectors: $\left|u_{1}\right\rangle,\left|u_{2}\right\rangle, \cdots,\left|u_{\infty r}\right\rangle\left(\left\{\mathcal{W}_{u}\right\}\right.$ for short) such that we can write

$$
|\psi(t)\rangle=\sum_{j} c_{j}(t)\left|u_{j}\right\rangle
$$

where $c_{j}(t)=\left\langle u_{j} \mid \psi(t)\right\rangle$ and $j=1,2, \cdots, \mathcal{\&}$. The mean value of $\Delta$ at time $t$ is $\langle\mathfrak{Q}\rangle(t)$ given by Equation (A3); in particular, if $|\psi(t)\rangle=\left|u_{j}\right\rangle$ the mean value of $\mathcal{A}$ is $\left\langle u_{j}|\mathscr{Q}| u_{j}\right\rangle$. Then, if Equations (A1) and (A2) are satisfied, we shall require the validity of

$$
\langle\psi(t)|\mathfrak{Q}| \psi(t)\rangle=\sum_{j}\left|c_{j}(t)\right|^{2}\left\langle u_{j}|\mathfrak{Q}| u_{j}\right\rangle
$$

for the state $|\psi(t)\rangle$ given by Equation (A4) may collapse to the vectors of the 
set $\left\{\alpha_{u}\right\}$, i.e. for jumps like $|\psi(t)\rangle \rightarrow\left|u_{1}\right\rangle$, or $|\psi(t)\rangle \rightarrow\left|u_{2}\right\rangle, \cdots$, etc., may occur [18] [19] [20]. In previous papers we have introduced this requirement as a postulate ensuring the statistical sense of conservation laws [18] [19] [20]. Needless to say that this postulate forbids projections of the state $|\psi(t)\rangle$ to the vectors of many sets $\left\{\alpha_{u}\right\}$.

If there is a unique set of $\propto<\geq 2$ orthonormal vectors: $\left|\varphi_{1}\right\rangle,\left|\varphi_{2}\right\rangle, \cdots,\left|\varphi_{\text {कr }}\right\rangle$ ( $\left\{\mathcal{\gamma}_{\varphi}\right\}$ for short) such that (I) the state of $\delta$ at time $t$ can be written

$$
|\psi(t)\rangle=\sum_{j} \gamma_{j}(t)\left|\varphi_{j}\right\rangle
$$

where $\gamma_{j}(t)=\left\langle\varphi_{j} \mid \psi(t)\right\rangle \neq 0$ for every $j=1,2, \cdots, \propto$; (II) at least ( $\left.\&-1\right)$ vectors belonging to the set $\left\{\mathcal{\gamma}_{\varphi}\right\}$ are eigenstates of the Hamiltonian $\mathcal{E}$ (i.e. stationary states); and (III) every self-adjoint operator $\mathscr{Q}$ for which Equations (A1) and (A2) are valid satisfies the relation

$$
\langle\psi(t)|\mathcal{Q}| \psi(t)\rangle=\sum_{j}\left|\gamma_{j}(t)\right|^{2}\left\langle\varphi_{j}|\mathcal{Q}| \varphi_{j}\right\rangle
$$

we shall say that $\left\{\delta_{\varphi}\right\}$ is the preferential set of $\delta$ in the state $|\psi(t)\rangle$ and the members of $\left\{\delta_{\varphi}\right\}$ will be called its preferential states. The preferential set depends on the system's state $|\psi(t)\rangle$. Note: Differing from the definition of preferential set previously introduced [18] [19] [20], the present one imposes the condition $\gamma_{j}(t)=\left\langle\varphi_{j} \mid \psi(t)\right\rangle \neq 0$ for every $j=1,2, \cdots$, \& .

The concept of preferential states plays a paramount role in SPA for $|\psi(t)\rangle$ can jump only to these states; if the system in the state $|\psi(t)\rangle$ does not have preferential states, the Schrödinger evolution follows.

\section{Appendix B: Some Examples}

(a) The simplest case is that where the Hamiltonian $\mathscr{E}$ is a complete set of compatible operators with entirely discrete spectrum. The eigenvalues will be denoted by $E_{n}$ where $n=1,2, \cdots$; and the corresponding eigenvectors by $\left|\phi_{n}\right\rangle$. The state vector of the physical system $\delta$ can be written

$$
|\psi(t)\rangle=\sum_{n} c_{n}(t)\left|\phi_{n}\right\rangle
$$

Since $\mathcal{E}$ is a constant of the motion, $|\psi(t)\rangle$ has the tendency to collapse to the eigenvectors $\left|\phi_{n}\right\rangle$. As the relation

$$
\langle\psi(t)|\mathcal{}| \psi(t)\rangle=\sum_{n}\left|c_{n}(t)\right|^{2}\left\langle\phi_{n}|\mathcal{E}| \phi_{n}\right\rangle
$$

is valid, the requirement (III) established in Appendix A is satisfied for every $|\psi(t)\rangle$. In the particular case where the state vector of $\delta$ at time $t_{1}$ is

$$
\left|\psi\left(t_{1}\right)\right\rangle=c_{j}\left(t_{1}\right)\left|\phi_{j}\right\rangle+c_{k}\left(t_{1}\right)\left|\phi_{k}\right\rangle
$$

where $c_{j}\left(t_{1}\right) \neq 0$ and $c_{k}\left(t_{1}\right) \neq 0$, conditions (I) and (II) of Appendix A are also fulfilled. Hence the preferential set of $\delta$ in the state $\left|\psi\left(t_{1}\right)\right\rangle$ is $\left\{\left|\phi_{j}\right\rangle,\left|\phi_{k}\right\rangle\right\}$, a subset of the set of stationary states. This is a general rule in case $\mathcal{E}$ is a constant of the motion and a complete set of compatible operators with discrete spectrum. 
(b) We assume that the operators $\mathscr{E}, \mathcal{A}$ and $\mathcal{B}$, respectively representing the physical quantities energy, $\triangle$ and $₫$ are constants of the motion and have discrete spectra. In addition, we suppose that the set $\{\mathscr{E}, \mathscr{Q}, \mathscr{B}\}$ is the unique complete set of compatible operators of the system. The eigenvectors of its common basis will be denoted by $\left|E_{j} a_{k} b_{l}\right\rangle$, where $E_{j}, a_{k}$ and $b_{l}$ are respectively the eigenvalues of $\mathcal{E}, \mathscr{Q}$ and $\mathcal{B}$, and $j=1,2, \cdots ; k=1,2, \cdots$; $l=1,2, \cdots$ The system's state has tendency to jump to the eigenvectors of $\mathscr{E}$, to the eigenvectors of $\mathscr{Q}$, and to the eigenvectors of $\mathcal{B}$. Since the relations

$$
\begin{aligned}
& \langle\psi(t)|\mathcal{E}| \psi(t)\rangle=\sum_{j k l}\left|c_{j k l}(t)\right|^{2}\left\langle E_{j} a_{k} b_{l}|\mathcal{E}| E_{j} a_{k} b_{l}\right\rangle \\
& \langle\psi(t)|\mathscr{Q}| \psi(t)\rangle=\sum_{j k l}\left|c_{j k l}(t)\right|^{2}\left\langle E_{j} a_{k} b_{l}|\Theta| E_{j} a_{k} b_{l}\right\rangle
\end{aligned}
$$

and

$$
\langle\psi(t)|\mathcal{B}| \psi(t)\rangle=\sum_{j k l}\left|c_{j k l}(t)\right|^{2}\left\langle E_{j} a_{k} b_{l}|\mathcal{B}| E_{j} a_{k} b_{l}\right\rangle
$$

are satisfied for

$$
|\psi(t)\rangle=\sum_{j k l} c_{j k l}(t)\left|E_{j} a_{k} b_{l}\right\rangle
$$

condition (III) stated in Appendix A is fulfilled for every state of the system. In the particular case where the state vector at $t_{0}$ is

$$
\left|\psi\left(t_{0}\right)\right\rangle=\gamma_{111}\left(t_{0}\right)\left|E_{1} a_{1} b_{1}\right\rangle+\gamma_{212}\left(t_{0}\right)\left|E_{2} a_{1} b_{2}\right\rangle
$$

where $\gamma_{111}\left(t_{0}\right) \neq 0$ and $\gamma_{212}\left(t_{0}\right) \neq 0$, it is easily verified that conditions (I) and (II) established in Appendix A are also satisfied. So the preferential set of $\delta$ in the state $\left|\psi\left(t_{0}\right)\right\rangle$ is $\left\{\left|E_{1} a_{1} b_{1}\right\rangle,\left|E_{2} a_{1} b_{2}\right\rangle\right\}$. As in the previous case, every preferential set is a subset of the set of stationary states.

(c) We assume, as in case (b), that $\mathcal{E}, \mathcal{A}$ and $\mathcal{B}$ are constants of the motion and have discrete spectra but $[\mathscr{Q}, \mathcal{B}] \neq 0$. Hence $\mathscr{Q}$ and $\mathscr{B}$ do not have a common basis. Let us suppose, however, that $\{\mathscr{E}, \mathscr{A}\}$ and $\{\mathscr{E}, \mathfrak{B}\}$ are two complete sets of compatible operators. In the basis of the former, every state can be written

$$
|\psi(t)\rangle=\sum_{j k} c_{j k}(t)\left|E_{j} a_{k}\right\rangle
$$

while in the basis of the latter we have

$$
|\psi(t)\rangle=\sum_{j l} d_{j l}(t)\left|E_{j} b_{l}\right\rangle
$$

Collapses to the vectors of the basis $\left\{\left|E_{j} a_{k}\right\rangle\right\}$ violate the statistical sense of the conservation of $₫$ while reductions to the vectors of the basis $\left\{\left|E_{j} b_{l}\right\rangle\right\}$ violate the statistical sense of the conservation of $\mathcal{A}$ [18]. By contrast, jumps to the normalized projection of $|\psi(t)\rangle$ into the eigensubspace of $\mathscr{E}$ corresponding to the eigenvalue $E_{j}$ are in compliance with both the statistical sense of the conservation of the physical quantity $A$ and the statistical sense of the conservation of $₫$ [18]. Also in this case every preferential set is a subset of the set of stationary states. 
Submit or recommend next manuscript to SCIRP and we will provide best service for you:

Accepting pre-submission inquiries through Email, Facebook, LinkedIn, Twitter, etc. A wide selection of journals (inclusive of 9 subjects, more than 200 journals)

Providing 24-hour high-quality service

User-friendly online submission system

Fair and swift peer-review system

Efficient typesetting and proofreading procedure

Display of the result of downloads and visits, as well as the number of cited articles Maximum dissemination of your research work

Submit your manuscript at: http://papersubmission.scirp.org/

Or contact jmp@scirp.org 